\title{
GLOBAL MANUFACTURING STRATEGY
}

\author{
Vedat Verter \\ University of Alberta
}

\section{Cemal Dincer Bilkent University}

\subsection{Introduction}

In response to the intensifying rivalry in many industries, firms are restructuring themselves to operate on a global basis. Globalization of a firm constitutes diversification of its operations to different countries so as to take advantage of the inefficiencies in the international product, factor, and capital markets. Global configuration of operations provides access to cheaper labor and raw materials, subsidized financing opportunities, and larger product markets. A global firm however, can achieve competitive advantages only if its geographically dispersed activities are effectively coordinated. That is global firms "must learn to operate as if the world were one large market - ignoring superficial regional and national differences" [759]. Although multinational companies have a long history, globalization of operations is a recent phenomenon. A typical multinational corporation consists of several strategic business units (SBUs) each functioning in a particular industry and serving a well-defined market segment. Each SBU, in turn, consists of several functional units such as purchasing, marketing, finance, personnel, $R \& D$, and manufacturing. Such a three-level hierarchy is depicted in Figure 1. Traditionally, multinational companies were operated as multidomestic corporations where each SBU is run as a domestic firm. What we are currently witnessing however, is an internationalization at the SBU level via the location of functional units at different countries. As a result of these developments, managers are facing new challenges such as, the need to incorporate the differentiating features of the international environment in decision making, and the difficulties of dealing with the increased organizational complexity due to globalization.

In this chapter, we will confine ourselves to the strategy planning problems at a specific functional unit in the global firm, i.e. manufacturing. The long term goals for manufacturing performance and the policies adopted 
FIGURE 1. A three-level corporate hierarchy

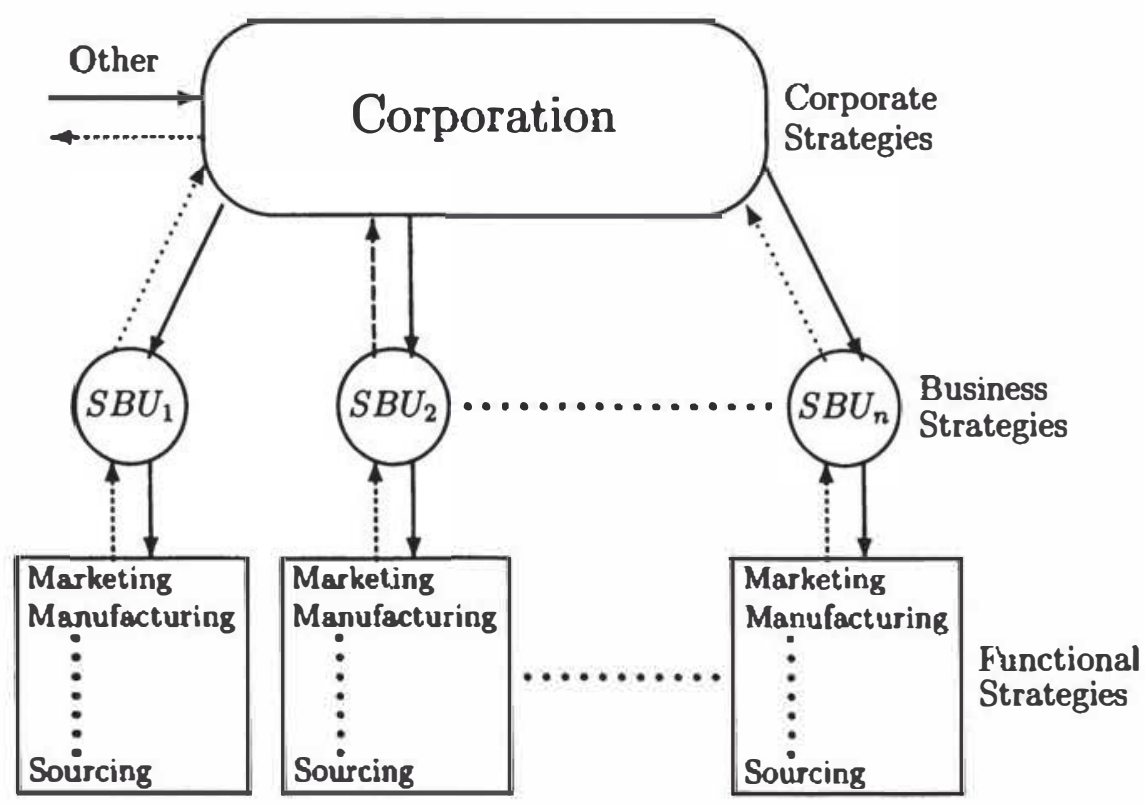

Investment

Return

to achieve those goals constitute manufacturing strategy of a firm. Cost, quality, delivery performance, and flexibility are the most common criteria to evaluate performance of a manufacturing system. It should be noted that firms are not in a position to choose between cost and quality or dependability and flexibility objectives. Empirical studies did not justify the existence of such tradeoffs suggested in early conceptual work on manufacturing strategy. In accordance with the intensive rivalry in global industries, innovativeness and time-based competition are also emerging as important manufacturing objectives. Policies that enable a firm to meet its long term goals comprise a collection of strategic decisions. Leong et al. [756] pointed out the consensus among several authors about the strategic decision areas for manufacturing. Manufacturing strategy decisions can be categorized as structural decisions that are associated with configuration of the manufacturing facilities, and infrastructural decisions that address the people and systems that run the manufacturing activity. Structural decisions include location of the manufacturing facilities, and the manufacturing technology to be adopted as well as the capacity to be built-in at each facility. The decisions concerning the linkages among the facilities that perform different 
stages of the production process, and the interactions of manufacturing facilities with suppliers and customers are also in this group. Infrastructural decisions are associated with production planning and control, quality control, workforce management, new product development, and performance measurement systems. Note that, manufacturing is an integral part of the firm, and therefore manufacturing strategy cannot be formed without considering its interactions with the business and corporate strategies as well as with other functional strategies as pointed out by Fine and Hax [403].

Production-distribution networks provide an effective tool in modeling manufacturing structure of a firm. A typical production-distribution network is depicted in Figure 2. Nodes of the network represent vendors, manufacturing facilities, distribution centers, warehouses, and customer zones whereas arcs represent the flow of items. In a global firm, nodes of the production-distribution network are located in different countries, and items flow across national boundaries. Each layer of the network in Figure 2 is called an echelon, and geographical dispersion of facilities usually results in a multi-echelon configuration. Another common feature of global manufacturing is the production and distribution of a variety of products to benefit from economies of scope. The multicommodity nature of international production-distribution networks is fostered by the differ ent needs of each national product market. Manufacturing strategy planning involves decisions regarding the long term changes in configuration of the production-distribution network of the firm. Facility location decisions are crucial in strategy planning, especially in globalization of the manufacturing activity. Availability and cost of production factors such as, qualified labor, reliable raw material and component supply, and manufacturing technology vary significantly among countries. Thus, within the international context, location of a facility interacts with its size, technology content, and product range in facilitating the achievement of strategy goals. Ignoring these interactions in locational decisions will presumably lead to a suboptimal manufacturing configuration in a global firm. We sug gest that global manufacturing strategy of the firm provides a framework for the facility location decisions.

The aim of this chapter is to provide a survey of the analytical models that are relevant to the facility location decisions in a global firm. This is an emerging field of research and most of the reviewed studies are far from capturing all of the characteristics of global manufacturing. Our criterion for including an analytical approach in this survey is that it should incorporate at least one of the characteristics of global manufacturing such as, multiple products, multiple echelons of manufacturing facilities, exchange rates, or price uncertainties. The remainder of this chapter is organized as follows. The second section briefly describes the requirements of the global manufacturing strategy planning process in setting the framework for locational decisions. The third section reviews the analytical models for the multicommodity, multi-echelon production-distribution system design 
FIGURE 2. Production-Distribution network

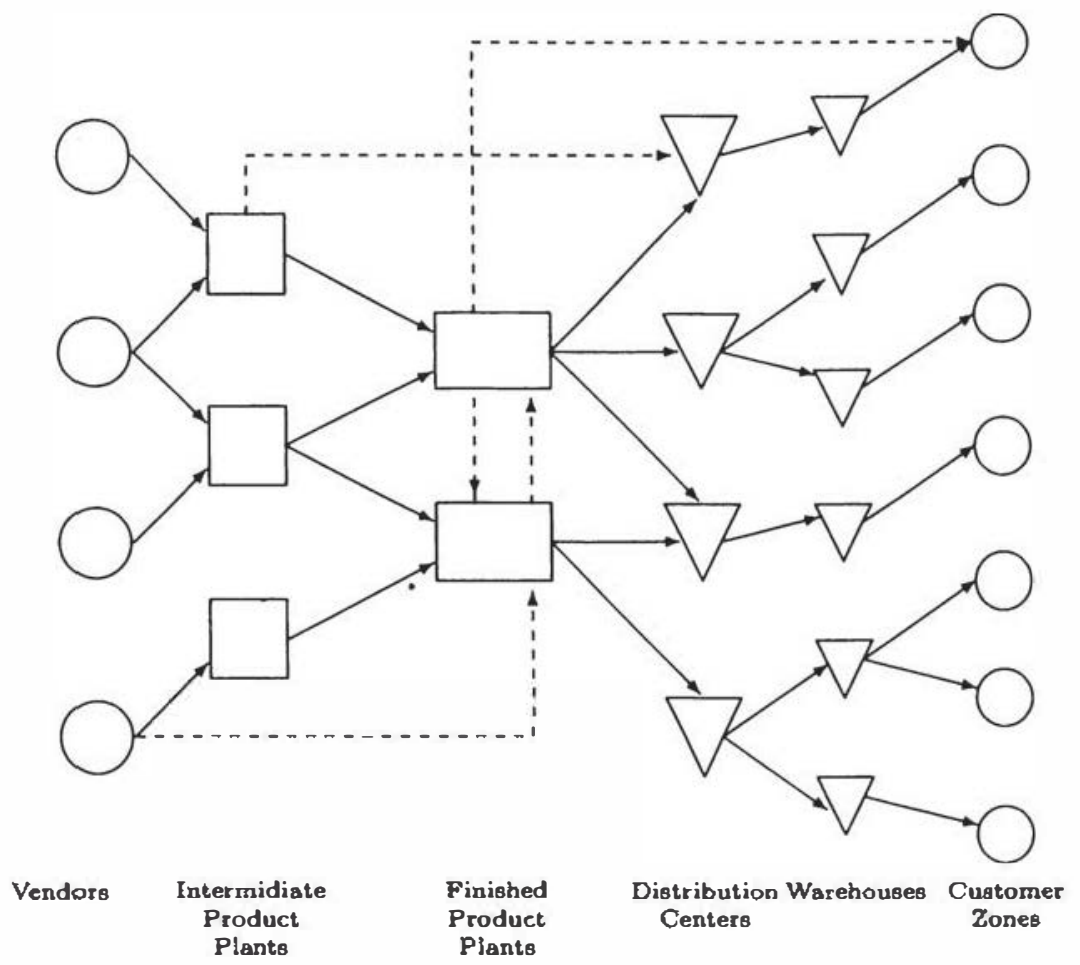

problem. Note that, although these models can also be used for strategy planning in domestic firms, they provide a sound basis for incorporating various features of the international environment. Thus, designing global manufacturing networks is usually perceived as an extension of the basic multicommodity, multi-echelon network design problem, as presented in the fourth section.

\subsection{Global Manufacturing Strategy Planning Process}

Access to low cost production input factors constitutes the earliest and most common motivation for global manufacturing. The international differentials in manufacturing costs however, are diminishing as pointed out by Flaherty [407]. Improvements in the transportation and communication industries however, foster the emergence of global products that can simultaneously be marketed in several countries. This enables the global 
firms to achieve scale economies in constructing their manufacturing facilities, and hence in their manufacturing technology investments. Proximity to customer zones, use of local technological resources, and pre-emption of competition via early-mover advantages are further strategic reasons for global manufacturing as observed by Ferdows [399]. Hitt et al. [575] pointed out that there is a positive correlation between global diversification of operations and firm performance.

There are several distinguishing features of the international environment which have to be taken into consideration for global manufacturing strategy planning. According to classical economic theory, the law of comparative advantages provides a basis for international transactions. That is, trade will be mutually advantageous if countries are relatively more efficient in producing different goods. Ideally, an international equilibrium will be attained when exports and imports of individual countries reach a balance in their own currency. Exchange rates among currencies provide the means for adjustment of the international disequilibrium. This is apparent from the following theorems on the relationships between exchange rates, interest rates and price levels: Purchasing Power Parity (PPP) states that exchange rates off-set the differences between national price levels of freely tradeable goods, and International Fisher Effect (IFE) states that exchange rates off-set the differences between interest rates for different currencies. These fundamental exchange rate relationships however, are based on some assumptions such as; perfect product markets (no transportation cost or time, no barriers to trade), perfect financial markets (all relevant information is reflected in prices, no taxes, no transaction costs, no controls), and future certainty. These assumptions are quite unrealistic in many cases, since product and capital markets are not "perfect" and the future is not known with certainty. Deviations from PPP exist due to lags in market responses, transportation costs, national differences in the price ratios of internationally traded goods to domestically traded goods, government interventions and risk expectations. Furhermore, deviations from IFE exist due to the availability of subsidized financing and differentials in corporate tax rates. In addition to the various arbitrage opportunities provided by these imperfections, uncertainties regarding future exchange rates, interest rates and price levels cause various types of risks the firms have to undertake when designing their manufacturing strategy. Distinguishing features of the international environment and their potential impacts on firm behavior are depicted in Table 12.1.

Despite growing importance of the globalization phenomenon, the review by Adam and Swamidass [9] showed that the international content of manufacturing activity is among the missing themes in manufacturing strategy literature. Recently, Miller and Roth [844] provided a taxonomy of the manufacturing strategies identified by a survey of 164 manufacturing companies in the United States. De Meyer (276) presented a similar empirical study for European manufacturers. In an earlier study, Ferdows et 
TABLE 12.1. Distinguishing Features of the International Environment

\begin{tabular}{|l|l|}
\hline \hline Phenomena & Effect \\
\hline \hline Deviations from PPP & goods arbitrage opportunity \\
Deviations from IFE & financial arbitrage opportunity \\
Price uncertainty & market risk \\
Exchange rate uncertainty & currency risk \\
Government interventions & political risk \\
Quotas, local content rules & constrain flow of items \\
Tariffs, duties & increase transaction costs \\
Cultural, taste differences & product tailoring \\
Language, skill differences & human factors management \\
\hline \hline
\end{tabular}

al. [400] compared the evolving global manufacturing strategies in United States, Europe and Japan. Although these empirical studies provide some insight about the current practice in global manufacturing, Skinner [1085] pointed out the lack of methodologies to identify the most appropriate manufacturing structure in achieving the manufacturing strategy objectives. A prevailing approach is due to Kotha and Orne [714] who extended the work of Hayes and Wheelwright [568,569]. They perceived the man ufacturing activity as a three dimensional structure, the dimensions being process structure complexity, product line complexity, and organizational scope. Factors leading to the high or low level of complexity at each of these dimensions are further identified, and analysed. In this context, manufacturing strategy constitutes a movement in the three dimensional space from a point that designates the current manufacturing structure to a target point implied by the strategy goals. Their work revealed the need that the product range and organizational structure of each manufacturing facility should be consistent with the target market segment and geographic scope of operations. Further, each plant should select and adopt the technology that is most appropriate in terms of its strategic role in the company. Ferdows [399] presented a scheme for matching the technology content of a manufacturing facility with the primary reason for its location in a foreign country. Although the work of Kotha and Orne [714) provided valuable guidance on how to achieve the fit between strategy and structure, their representation of the manufacturing activity remains quite descriptive.

The aim in manufacturing strategy planning is achievement of long-term sustainable competitive advantages over the rivals via configuration and co. ordination of the firm's production-distribution system. Global firms have facilities located in different countries. Note that there are differences between countries in terms of cost and quality of the available production factors as well as institutional and cultural infrastructure. Thus, in many industries, it is possible to identify some countries that provide compare- 
tive advantages for performing ccrtain stages of the manufacturing activity. Kogut [706] described the interplay of the firm specific competitive advantages and the location specific comparative advantages for global firms. One of the primary requirements of global manufacturing strategy planning process is the ability to take advantage of the interrelations between competitive and comparative advantages. A critical factor in global competitiveness is the comparative advantages of the home base for global firm's activities in the relevant industry. A home base is the country which reaps the profits and which is usually where the majority of production and management takes place. Porter [970] provided a paradigm to assess the attractiveness of a nation as a home base for an industry, and identified the determinants of national advantage: factor conditions; demand conditions; related and supporting industries; and firm strategy, structure, and rivalry. Government intervention and uncertainty are perceived as the factors influencing these determinants. This theory is implemented through the analysis of a selection of industries in each of the following countries: Denmark, Germany, Italy, Japan, Korea, Singapore, Sweden, Switzerland, United Kingdom and the United States. Kogut [707] pointed out the importance of creating operational flexibility within the corporation in order to benefit from being global. Operational flexibility provides the capability to explore arbitrage and leverage opportunities. Arbitrage opportunities are associated with global configuration and include; production shifting in response to changing circumstances in factor markets, information arbitrage, tax minimization via transfer pricing, and financial arbitrage via subsidized loans. Leverage opportunities arise from global coordination and may be a hedge against the political risk associated with international investments.

Global coordination of the manufacturing activity is equally important with the configurational decisions in global manufacturing strategy planning. The inbound and outbound logistics activities assure the coordination of material flow. Fawcett [396] stressed the importance of strategic logistics in global manufacturing success, and presented a review of the literature on logistics. The sharing and use of information throughout the global network is essential in managing global manufacturing. De Meyer and Ferdows [277] presented empirical evidence suggesting that integration of information systems within manufacturing, and between manuf acturing and other functions is a growing concern in Europe, North America and Japan. Flaherty [406] described the role of support activities in coordination of the manufacturing and technology development taking place in internationally dispersed facilities. Global coordination of the manufacturing activity also involves allocating responsibility to the dispersed facilities and aligning their technology development efforts which need to be supported by the organizational culture as pointed out by Misterek et al. [857]. In order to assure success at the firm level however, the manufacturing activity has to be coordinated with the other functional units. The cross-functional coordination of manufacturing and marketing is emphasized in the work of Hill 
[573].

This section is intended to be a brief account of the factors that differentiate global manufacturing from the manufacturing activity in a domestic firm. Naturally, these factors should be incorporated in the manufacturing strategy of a global firm. Thus, requirements of the global manufacturing strategy planning process constitutes a framework for facility location decisions. An analytical approach for locational decisions can facilitate the achievement of strategy objectives to the extent that it incorporates the interaction of configurational decisions and the characteristics of the international environment. Having set the criteria to evaluate the relevance of an analytical model for facility location decisions in a global firm, we now turn to review the state-of-the-art. It should be noted that the above discussion is confined to the effects of globalization on the manufacturing activity. The changes in firm structure due to globalization, such as joint ventures, strategic alliances, franchising and licensing are out of the scope of this chapter. The interested reader however, is referred to the recent bibliography in [742] and to the books edited by Porter, [969] Ferdows, [398] and Sheth and Eshghi [1072].

\subsection{The Production-Distribution System Design Problem}

Given a set of markets to be served, the sets of alternative facility locations and technologies for each stage of the production and distribution process, and a set of alternative vendors the production-distribution system design problem involves decisions regarding the number and location of facilities in each echelon, the product range, capacity and technology content of each facility as well as the flow of items through the network so as to minimize the total cost of serving the clients. The cost items involved in designing a production-distribution network are the fixed costs of facility construction, the variable costs of technology acquisition and operation as a function of the capacity to be built-in at each facility, and the inbound and outbound logistics costs. Considerable progress has been achieved in the development of tools for solving the relatively simpler single commodity single-echelon production-distribution system design problems as presented by Verter and Dincer [1163). The design problem of a global firm however, would normally have a multicommodity, multi-echelon structure on which the literature is rather sparse. An earlier review of the facility location models for distribution planning problems is due to Aikens [13]. In this section we review the literature pertaining to the development of analytical techniques for solving the production-distribution system design problem stated above in its most general form.

The uncapacitated facility location problem (UFLP) that involves locat- 
ing an undetermined number of facilities to minimize the fixed setup costs plus linear variable costs of serving clients constitutes a building block in solving the multicommodity, multi-echelon design problems. The multicommodity uncapacitated plant location problem (MUPLP) is a generalization of the UFLP where multiple products are required by the clients, and an additional fixed cost is incurred if an open plant is equipped to manufacture a particular product. One of the earliest works on multicommodity problems is due to Warszawski [1174] where each plant is restricted to produce a single commodity. Warszawski [1174] devised a heuristic algorithm whereas, Neebe and Khumawala [885] suggested a branch and bound procedure for this class of problems. Klincewicz, Luss and Rosenberg [704] provided an optimal and various heuristic branch-and-bound procedures for solving the MUPLP. They suggested decomposition of the nodal problems into sep. arate UFLPs each associated with a product, for the calculation of the lower bounds. Akinc [14] analyzed the capacitated version of the problem where the size of a plant to be established is bounded above. He presented a branch-and-bound algorithm which constitutes an extension of the Akinc and Khumawala [15] algorithm for the capacitated facility location prob. lem to the multicommodity setting. Klincewicz and Luss [703] developed a dual-based algorithm for solving the MUPLP. Their algorithm is inspired by the dual-based approach of Erlenkotter [390] for the UFLP. Erlenkotter's dual ascent and dual adjustment procedures are extended to generate a good feasible solution to the dual of the linear programming relaxation of MUPLP. These procedures are incorporated in a branch-and-bound algorithm for providing an optimal solution to the MUPLP. Klincewicz and Luss [703] reported solving extensions of the 25 plant locations 50 markets Kuehn and Hamburger [721] problem as well as a set of random MUPLP's. The computational performance of their algorithm is encouraging since the set of sixteen MUPLPs (based on the Kuehn and Hamburger problem) consisting of 3,5 , and 10 product problems, required only 18.42 seconds on the average on an Amdahl 470/V8 computer.

The two echelon uncapacitated facility location problem (TUFLP) is a generalization of the UFLP where the commodity is processed at two echelons of facilities before being served to the clients. Kaufman, Eede and Hansen [680] suggested a generalization of Efroymson and Ray's [365] UFLP algorithm, for simultaneous location of uncapacitated single-commodity plants and warehouses. It is also possible to utilize their model for locating two echelons of warehouses given the locations of production facilities. Ro and Tcha [1009] provided a branch-and-bound procedure for solving the TUFLP with some side constraints. Their work extends the Efroymson and Ray [365] and Khumawala [693] algorithms to the TUFLP. Tcha and Lee [1122] suggested a branch-and-bound approach for the multiechelon UFLP in which the dual ascent procedure of Erlenkotter [390] is utilized. Their computational experiments however, focus on the TUFLP. $\mathrm{Gao}$ and Robinson [442) devised a dual-based optimization procedure for 
solving the TUFLP. Erlenkotter's dual ascent and dual adjustment procedures arc extended to generate a good feasible solution to the dual of the linear programming relaxation of TUFLP. This solution provides a lower bound on the value of the optimal solution to TUFLP. Further, based on the dual solution, a feasible primal solution is constructed using the complementary slackness conditions. As in Erlenkotter [390] these procedures are incorporated in a branch-an d-bound algorithm for providing an optimal solution to the TUFLP. Gao and Robinson [442] reported solving TUFLPs with 25 facility locations at each echelon and 35 markets in 2.4 seconds on a CDC Cyber 170/855 computer.

Capacitated, multiconumodity, multi-echelon formulations are mostly focused on location of a single echelon of warehouses on the basis of existing production facilities. Elson [377\} presented one of the earliest studies where the availability of management options to expand existing distribution centers (DCs) in addition to opening new ones is also incorporated. Proposed optimization procedure however, decomposes the commodity flows into the plant-to-DC and the DC-to-customer portions. Geoffrion and Graves [454] provided the most influential work on the multicommodity productiondistribution system design problem. Given a set of production plants each with known capacity, the authors were concerned with locating a single echelon of DCs and assigning those DCs to customer zones in order to satisfy the demand. The model formulation is as follows:

$$
\begin{array}{lc}
\text { Minimize } z=\sum_{p, i, d, j} c_{p i d j} X_{p i d j}+\sum_{d}\left[F_{d} Y_{d}+v_{d} \sum_{p, j} D_{p j} Z_{d j}\right] \\
\text { subject to } \\
\sum_{d, j} X_{p i d j} \leq S_{p i}, \quad \forall p, i, \\
\sum_{i} X_{p i d j}=D_{p j} Z_{d i}, \quad \forall p, d, j, \\
\sum_{d} Z_{d j}=1, \quad \forall j, \\
\underline{V}_{d} Y_{d} \leq \sum_{p, j} D_{p j} Z_{d j} \leq \bar{V}_{d} Y_{d,}, \quad \forall d,
\end{array}
$$

Linear configuration constraints on $Y$ and/or $Z$,

$$
\begin{gathered}
Y_{d,}, \quad Z_{d j}, \in\{0,1\}, \quad \forall d, j, \\
X_{\text {pidi }} \geq 0, \quad \forall p, i, d, j,
\end{gathered}
$$

where

$p=$ index for commodities,

$i=$ index for the existing production plants,

$d=$ index for potential DC sites,

$j=$ index for customer zones (CZs),

$S_{p i}=$ production capacity of plant $i$ for commodity $p$,

$D_{p j}=$ demand for commodity $p$ in $\mathrm{CZ} j$, 
$\underline{V}_{d}, \nabla_{d}=$ minimum, maximum allowed annual throughput for DC $d$,

$F_{d}=$ annualized fixed setup cost of opening DC $d$,

$v_{d}=$ variable unit cost of throughput for DC $d$

$c_{p i d j}=$ unit cost of producing and shipping commodity $p$ from plant $i$ to $\mathrm{CZ} j$ through $\mathrm{DC} d$.

The decision variables are:

$X_{\text {pid }}=$ amount of commodity $p$ produced and shipped from plant $i$ to $\mathrm{CZ} j$ through DC $d$,

$Y_{d}=1$ if DC $d$ is opened, 0 otherwise,

$Z_{d j}=1$ if DC $d$ serves $\mathrm{CZ} j, 0$ otherwise.

Constraints (12.2) are supply constraints and (12.3) ensure that demand from each $\mathrm{CZ}$ will only be satisfied by a $\mathrm{DC}$ assigned to serve that $\mathrm{CZ}$. The single-souncing of CZs by DCs is imposed by constraints (12.4). That is the model suggests construction of a dominant $\mathrm{DC}$ for each $\mathrm{CZ}$ which fully serves the demand. Constraints (12.5) keep the total annual throughput of each DC between the required limits. They also enforce that a closed DC cannot be assigned to serve a CZ. Linear configuration constraints (12.6) allow representation of managerial requirements about the selection of DC sites and the DC-CZ assignments in the model, The objective is to minimize the sum of total production, transportation, DC construction and operation costs. Geoffrion and Graves [454] adopted a variant of the Benders [75] decomposition that solves the master problem as a feasibility problem. This is primarily in order not to waste effort solving a master problem to optimality when there are only a few Benders cuts to represent the subproblem at the earlier iterations. Their algorithm also describes how to synthesize the dual solutions to the single-commodity transportation subsubproblems to obtain dual solutions to the multicommodity transportation subproblem. The authors reported application of the solution technique to a real problem for a major food firm with 17 commodity classes, 14 plants, 45 possible DC sites, and $121 \mathrm{CZs}$. They also mentioned another large scale application for a major manufacturer of hospital supplies.

Moon [863] extended the problem formulation to incorporate the nonlinearities in DC throughput costs due to economies of scale. He presented an application of the Generalized Benders Decomposition devised by $\mathrm{Ge}$ off rion [449] to the nonlinear production-distribution system design problem. Approximate dual prices are generated by solving linear (instead of concave) subproblems which are then adjusted to better represent the concavity in throughput costs. These adjusted dual prices are incorporated in the Benders cuts. The computational results are reported to be encour aging. Van Roy [1159] presented an extended application of the Geoffrion 
and Graves [454] model for multi-level production-distribution planning and transportation fleet optimization. The problem belongs to a liquified petroleum gas company with 2 commodities (propane and butane), 2 refineries, 10 potential bottling plant locations, 40 potential depot locations, 40 potential breakpoints (transporters' home sites), and 200 customer regions. Location and capacity expansion decisions associated with the bottling plants, depots and breakpoints are given together with the decisions concerning the transportation fleet size, and the transportation shift systems and schedules. Note that, this problem requires optimization of the location decisions regarding three echelons of the production-distribution system compared to Geoffrion and Graves [454] concerned with the location of a single-echelon of facilities. The problem was solved using a matrix generator for network-like problems and MPSARX [1160], a general-purpose mathematical programming software system augmented with automatic reformulation and cut generation features.

Cohen, Lee and Moon [230] presented an integrated model for productiondistribution system design as an implementation of the manufacturing strategy paradigm suggested in Cohen and Lee [228]. Production plant and DC locations, DC-CZ assignments, and flow of raw materials, intermediate and finished products through the system are simultaneously pro. vided so as to minimize the sum of plant / DC construction and operation costs, raw material purchase costs, and transportation costs. Constraints ensure that the number of open plants, production volume at each plant, and DC throughput levels are within their upper and lower limits, and certain production plants and DCs are fixed open as a managerial policy. Customer demand for each product need to be fully satisfied taking into account the production capacity and the raw material supply constraints. Cohen, Lee and Moon [230] made a special effort to capture the scale and scope economies in production costs. The base level production costs at each plant are adjusted via a production cost multiplier which is a function of the capacity utilization rate and the number of products produced. Thus, the model is a large scale mixed-integer mathematical program with a nonlinear objective function. The authors devised an iterative solution procedure controlled by a model hierarchy. The algorithm requires an initial plant configuration to be provided. Then, a DROP/ADD heuristic is utilized to generate a new plant configuration with either one less or one more plant. The current DC configuration is taken as input and initial DC-CZ assignments are either carried out by an assignment heuristic or provided by management. The first submodel is a linearly constrained nonlinear mathematical program that deals with the material flow through the network, and the product range at each facility. A simplex based algorithm is suggested for solving this subproblem. Submodel 1 provides plant pro. duction capacities as an input to the submodel 2 which is a distribution system design problem solved by the Geoffrion and Graves [454] procedure. Submodel 2 provides new DC configuration and DC-CZ assignments for the 
next iteration of submodel 1 . The DROP/ADD heuristic is activated for a new plant configuration upon convergence of the subproblem iterations. Cohen and Lee [228] reported the use of their model in the consulting practice of Booz Allen and Hamilton Inc.

Cohen and Moon [231] employed the model described above to investigate the impact of production scale economies, manufacturing complexity and transportation costs on production-distribution systems. They analyzed the behavior of optimal solutions in response to variations in the input parameters of the production-distribution system design problem. It has been observed that economies of scale and scope as well as transportation costs can significantly affect the system structure. Recently, Cohen and Moon [232] presented a plant loading model with economies of scale and scope. The plant loading problem takes the configuration of plants and DCs, and the DC-CZ assignments given, in order to optimize the product range at each facility and the flow of materials through the productiondistribution system. Note that, this problem corresponds to the first subproblem of Cohen, Lee and Moon [230]. In [232] cost of complexity is captured via a fixed cost of assigning a product line to a plant They represented economies of scale by the aid of a piecewise linear concave production cost function compared to the nonlinear representation in Cohen, Lee and Moon (230). A variant of Benders decomposition is suggested for solving this plant loading problem.

Defining the production-distribution system design problem at the outset enables us to provide an assessment of the state-of-the-art on multicommodity, multiechelon networks. It is observed that an overwhelming majority of the prevailing methods for designing such systems focus on the optimization of location and allocation decisions ignoring other dimensions of manufacturing structure i.e. capacity, technology content, and product range of the manufacturing facilities and their vertical integration. The capacity acquisition costs are not incorporated in the existing models which implies an implicit assumption that they would be the same at all sites. Since this assumption is not valid in the international context, the information provided by these models regarding the size of a new facility might be far from optimal. Although, it is possible to include the capacity acquisition costs in fixed setup costs in the capacitated models, it is highly likely that such predetermined sizes for the new facilities would be sub. optimal. Further, there exist a variety of manufacturing technologies for each product that can be adopted in providing the capacity to be built-in a new plant. There is a vast literature on the analysis of investments in manufacturing technology such as $[402,974]$. Incorporation of the technology selection decisions in production-distribution system design models would lead to a significant improvement in their capability to assist strategic decision making. Modeling technology content of each facility in the network would also allow for addressing production flexibility, quality, and delivery performance, as well as costs in the network design process. 


\subsection{Designing International Production-Distribution Systems}

The previous section enables the reader to trace the development of methods for production-distribution system design and their validation through real life applications. These models allow for the incorporation of multiple echelons of facilities, multiple commodities, and the nonlinearities due to economies of scale and scope that are inherent in international networks, and hence provide valuable insights in designing such systems. Note that however, the analytical approaches mentioned above are confined to a cost minimization objective. This creates a deficiency in dealing with the uncertainties associated with product markets which are crucial in the international context. A significant line of research focused on the international plant location problem (IPLP) to remedy this weakness. Unfortunately, the improvements in the incorporation of uncertainty are offset by the fact that IPLP addresses a very simplistic (single commodity, single echelon) production-distribution system.

IPLP constitutes a challenging version of the UFLP within the interna tional context, and is stochastic by nature due to the randomness in price and exchange rate movements. National governments provide subsidized financing (as well as low tax rates) to attract multinational companies to locate production plants in their country. Multinational companies on the other hand, use foreign financing packages to hedge against international price and exchange rate fluctuations. Thus, financing decisions are an integral part of IPLP due to risk reduction strategies as well as locational incentives via subsidized interest rates. The pioneering work in modeling the interaction between international location and financing decisions is due to Hodder and Jucker [577]. That model however, is restricted to a deterministic setting. Hodder and Jucker [578] extended their previous work to incorporate uncertainty, ignoring financing decisions. They presented a single period model where a multinational company is assumed to be a mean-variance decision maker in terms of the after-tax profits. They modeled the random deviations from PPP via a single factor price generating mechanism as follows:

$$
P_{j} e_{1 j}=b_{j}\left(P_{1}+\epsilon_{j}\right),
$$

where

$P_{j}=$ the price at market $j$,

$e_{1 j}=$ the units of the numeraire currency per unit of currency $j$,

$b_{j}=$ market adjustment parameter

$P_{1}=$ the random price in the home country with mean $\overline{P_{1}}$ and variance $\sigma_{p}^{2}$, and 


$$
\epsilon_{j} \sim N\left(0, \sigma_{j}^{2}\right), \quad \operatorname{cov}\left(\epsilon_{j}, \epsilon_{k}\right)=0 \forall j, \forall k \neq j .
$$

Note that, although price uncertainty is explicitly taken into account, the incorporation of exchange rate uncertainty is rather implicit. Hodder and Dincer [576] developed a model for simultaneous analysis of the interna tional location and financing decisions. The mixed integer program has a quadratic objective function due to adoption of the mean-variance frame. work. The authors suggested a multifactor approach to diagonalize the variance-covariance matrix in the objective function. This results in a considerable reduction in the computational difficulty of solving IPLP. Recently, Min [848] suggested a chance-constrained goal programming model in order to incorporate the presence of dynamism and multiple objectives in the locational decisions of multinational firms. Although far from cap. turing the total complexity, international plant location methods provide a viable building block in designing international production-distribution systems.

Pomper [964] provided one of the earliest studies on international investment planning. He proposed a model to assist management in the evaluation of alternative manufacturing policies on a global basis. The model prescribes the optimal time-phasing of the location, technology and capacity investments as well as the optimal flow of materials throughout the future network. Pomper [964] analyzed the single-commodity, single production stage firms. He assumed that the multicommodity, multi-echelon structures can be decomposed into these easier to handle type of elements. Uncertainty in the environment is modeled by an uncertainty tree to represent the time-phased relationships among the environmental scenarios each occur with a certain probability conditional to the previous state of the environment. The expected present value of consolidated cash flow is maximized. Financial decisions are not considered although, Pomper [964] accepted that the international financial markets are not perfect. Economies of scale in production costs and in investment costs associated with the alternative technologies are approximated via fxed-charge linear functions. Dynamic programming is used to model and solve the international investment problem where a manufacturing state is defined to be the number of plants of each technology in each country. An alternative mixed-integer programming formulation of the problem is also presented which is claimed to be superior in large scale applications. Pomper [964] reported an applica tion of his model to a mature agricultural chemical product of a US-based multinational chemical company.

Kendrick and Stoutjesdijk [692] devised an investment project evaluation model. The single-country based firms are analyzed taking into account their international activities such as imports and exports. Their model can be conceived as a manufacturing strategy planning tool since the chosen investments constitute means to implement the manuf acturing policies. Decisions prescribed by the multiproduct, multiperiod model are; increments 
to the capacities of production units, shipments from plants to markets and among plants, exports, imports, domestic purchases of production factors, and by-product sales. The only set of integer variables in the mathematical program represents the capacity expansion decisions. Economies of scale in capacity acquisition is represented via a fixed-charge linear approximation. A two-stage production structure is incorporated and the future is assumed to be known with certainty. Net present costs are minimized to satisfy the demand by upgrading the current system via capacity expansion investments. Kendrick and Stoutjesdijk [692] suggested usage of the general-purpose integer programming softwares for solving their model.

Cohen, Fisher and Jaikumar [227] proposed a normative framework for strategic management of the international production-distribution systems. The firm's product range, production plant locations, capacities and production technologies are taken as given and the raw material sourcing, pro. duction, and market supply decisions are optimized. The multicommodity, multiperiod model seeks to maximize the net present value of the aftertax profits in the numeraire currency of the firm. Many of the international issues are incorporated such as duties and tariffs, currency exchange rates, differences in corporate tax rates in each country, market penetration strategies, and local content rules. Economies of scale in raw material purchasing is represented by the availability of a set of vendor contract options. There are fixed costs of plant loading which can be interpreted as costs of complexity. Furthermore, the fixed plant loading costs enable representation of the economies of scale in production via several "pseudoproducts" corresponding to the various cost rates associated with different levels of production. Production is assumed to have a single-stage structure and production plants are modeled to have capacity limits both in terms of the overall product range and on a per product basis. The Cohen, Fisher and Jaikumar [227] model is a mixed- integer nonlinear program. Nonlinearity in the objective function is caused by the co-existence of the $\hat{f}$ nancial decisions namely, transfer prices and overhead allocations together with the operational decisions. Hence, the authors suggested a hierarchical solution procedure. First step involves optimization of the operational variables concerning vendor contract selection, plant loading, purchasing, production, and market supply decisions on the basis of a fixed level of the financial variables. Second step solves for the optimal values of the financial variables, given optimal levels of the operational variables provided by step 1. This provides input for the next iteration of the first step. Cohen, Fisher and Jaikumar [227] suggested adoption of a mean-variance framework for incorporation of the price and exchange rate uncertainty in the international markets. The authors also suggested utilization of transfer prices for tax minimization purposes as well as for country-decomposed implementation of global manufacturing strategies.

Cohen and Lee [229] reported application of a variant of the Cohen, Fisher and Jaikumar (227) model in a multinational company manufac- 
turing personal computers. In the Cohen and Lee [229] study the product structure is modeled to include major components, subassemblies, and finished products. Obviously this increases the tradeoff capability. However, it should be noted that the model is deterministic and single-period which may partly offset the above enhancement. Cohen and Lee [229] perceived global manufacturing strategy as a collection of component strategies which are designed at various echelons of the production-distribution system. Component strategies are associated with the raw material sourcing, plant charter, and distribution/market supply activities. Firms have policy options for each of the component strategies. Various combinations of these policy options constitute the global policy options for the firm. The Cohen and Lee [229] model is essentially a strategy evaluation model since provision of the set of available global policy options is required by the solution procedure. A particular global policy option is translated into the struc turcal decisions of the model in an interactive manner. That is values of the indicator variables are fixed in order to evaluate the global policy option under consideration. Cohen and Lee [229] specified the following zero-one decision variables:

- Assignment of finished products and subassemblies to plants,

- Assignment of vendors to plants for each major component and sub. assembly,

- Assignment of vendors to DCs for each finished product that is sourced directly from vendors to DCs,

- Assignment of supply links from plants to DCs and from DCs to CZs,

- Transfer pricing policies for assigning transportation costs for intermediate and finished goods from one plant to another,

- Transfer pricing policies for assigning transportation costs of finished goods from plants to DCs,

- Determination of whether demands from a market region are to be satisfied.

Then, the remaining problem is a large scale linear program which involves material flow decision variables that denote the quantities of major components, subassemblies and finished products transported through the production-distribution system, and production decision variables that denote the quantities of items manufactured at the plants.

Recently, Huchzermeier [602] presented a model for global manufacturing strategy planning under exchange rate uncertainty. He suggested a multinomial approximation to the stochastic exchange rate process. A stochastic dynamic programming formulation is developed for evaluation of the global manufacturing strategy options. An option $O_{t}$ defines all operational and 
financial policies together with the structure of the production-distribution system at period $t$. State of the firm at the beginning of period $t$ is deter mined uy the current realization $i$ of the exchange rates denoted by vector $e_{0 t}^{i}$, and $O_{t-1}$. Hence, the recursion function is:

$$
V_{t}^{\prime}\left(e_{0_{t}}^{i}, O_{t-1}\right)=\max _{O_{t} \in \Omega_{t}}\left\{P_{t}\left(e_{O_{t}}^{i}, O_{t-1}, O_{t}\right)+\Phi_{t} \sum_{j} \pi_{i} j V_{t+1}\left(e_{0, t+1}^{j}, O_{t}\right)\right\}
$$

where

$V_{t}()=$. discounted value of the firm at period $t$, given the adoption of $O_{t-1}$ and the realization of $e_{0 t}^{i}$,

$\Omega_{t}=$ set of available global manufacturing strategy options at period $t$,

$\Phi_{t}=$ risk adjusted discount factor for period $t$,

$\pi_{i j}=$ the stationary transition probability from exchange rate realization vector $i$ to exchange rate realization vector $j$.

The profit at period $t$ is:

$$
P_{t}\left(e_{0 t}^{i}, O_{t-1}, O_{t}\right)=S P_{t}\left(e_{0 t}^{i}, O_{t}\right)-\delta\left(O_{t-1}, O_{t}\right),
$$

where

$$
\begin{aligned}
& S P_{t}(.)=\text { expected global after }- \text { tax profits for operating under } O_{t} \text { and } \\
& \mathrm{e}_{0 \ell}^{i}, \\
& \delta(.)=\text { switching cost from } O_{t-1} \text { to } O_{t} .
\end{aligned}
$$

At each period, the subproblem $S P_{t}$ is formulated as a stochastic pro. gram with recourse in order to also incorporate the demand uncertainty. The subproblem is essentially a constrained resource allocation problem and constitutes a variant of the Cohen and Lee [229] model. Huchzermeier [602] suggested a hierarchical procedure for solving the integrated model. Computational tractability however, decreases as the number of exchange rate processes and the number of demand scenarios increase.

Although, designing multicommodity, multi-echelon production-distribution networks attracted the attention of researchers for two decades, the studies that extend the basic design problem to incorporate the distinguishing features of international environment are not that many. It is observed that the most recent analytical methods for international production-distribution system design are developed for evaluation of alternative manufacturing configurations. Thus, the strategy design process is confined to the strategy options envisaged by management. This means that identification of the optimum configuration is conditional to its provision by the management as a viable strategy alternative. Given the size and complexity of 
the arising mathematical programs for designing international networks, the loss of computational tractability when such a model is treated in its entirety is quite natural. However, the alternative manuf acturing confgurations for a global firm proliferate, and hence generation of strategy options for manufacturing structure constitutes a problem by itself. Therefore, research is needed on the development of solution techniques that will enhance the optimization capability of international production-distribution system design methods in terms of the structural decision variables.

\subsection{Concluding Comments}

The prevailing analytical approaches for location of manufacturing facilities in a global firm can be classified into two broad categories:

i) Strategy Evaluation Models: These models are comprehensive in nature, and usually incorporate some of the features of international environment. Specification of a set of alternative manufacturing configurations is required. Each element of this set is represented by fixing values of the associated structural (integer) variables in the model. The remaining model in the (continuous) flow variables can then be used to evaluate the manuf acturing configuration under consideration.

ii) Strategy Generation Models: These models focus on the relatively simpler basic design problems and the IPLP. The structural and material flow decisions are provided simultaneously. It is however, still quite cumbersome to provide exact solutions to many of the arising mathematical programs which necessitates development of heuristic algorithms in many cases.

At this stage, the methodology for international production-distribution system design is in need of several enhancements to better capture the dynamics of global manufacturing strategy planning. First, the design techniques should be improved to address all the relevant competitive priorities such as quality, delivery performance and flexibility rather than focusing on cost as a predominant objective. Incorporation of the technology content of each facility in the multicommodity, multi-echelon network models would be a significant contribution toward that direction. Second, the design process should also address the policies regarding coordination of internationally dispersed facilities that would lead to global competitive advantage, not just the configurational decisions alone. Third, it should be realized that a production-distribution network represents only the manufacturing activity of the firm and its linkages with the buyers and suppliers. A comprehensive model of global competition however, requires incorporation of actions of the firm's current and potential competitors as well as gover n- 
ment interventions which may require a game-theoretic setting. Finally, strategy generation capability of the models for international productiondistribution system design needs to be improved via the development of new approaches to optimize the structural decisions. 